RIKEN-TH-171

\title{
Exploring Vacuum Structure around Identity-Based Solutions
}

\author{
Isao KISHIMOTO $^{1 *}$ and Tomohiko TAKAHASHI ${ }^{2 \dagger}$ \\ ${ }^{1}$ Theoretical Physics Laboratory, RIKEN, \\ Wako 351-0198, Japan \\ 2 Department of Physics, Nara Women's University, \\ Nara 630-8506, Japan
}

October, 2009

\begin{abstract}
We explore the vacuum structure in bosonic open string field theory expanded around an identity-based solution parameterized by $a(\geq-1 / 2)$. Analyzing the expanded theory using level truncation approximation up to level 20, we find that the theory has the tachyon vacuum solution for $a>-1 / 2$. We also find that, at $a=-1 / 2$, there exists an unstable vacuum solution in the expanded theory and the solution is expected to be the perturbative open string vacuum. These results reasonably support the expectation that the identity-based solution is a trivial pure gauge configuration for $a>-1 / 2$, but it can be regarded as the tachyon vacuum solution at $a=-1 / 2$.
\end{abstract}

\footnotetext{
*E-mail address: ikishimo@riken.jp

${ }^{\dagger}$ E-mail address: tomo@asuka.phys.nara-wu.ac.jp
} 


\section{Introduction}

Bosonic open string field theory (SFT) possesses classical solutions describing a tachyon vacuum where D-branes with attached open strings completely annihilate. A numerical tachyon vacuum solution was first constructed using level truncation approximation in the Siegel gauge [1. Then, an analytic classical solution has been constructed by Schnabl [2] and it has been found that the solution has several properties of the tachyon vacuum. The vacuum energy of the nontrivial analytic solution exactly cancels the D-brane tension, and the cohomology of the kinetic operator around the vacuum is trivial 3 .

In SFT, there is another type of analytic solution that was constructed earlier on the basis of the identity string field instead of wedge states used in Schnabl's solution [4]1] Interestingly, an identity-based solution discussed in Refs. 4, 6, 7, 8, has some properties of the tachyon vacuum. Moreover, for the identity-based solution, there is a possibility of understanding the existence of closed strings on the tachyon vacuum.

In $\S 2$, we will briefly summarize the identity-based solution. In $\S 3$ and $\S 4$, we will present new results for vacuum structure around the identity-based solution [5]. Then, we propose that the solution can be regarded as the analytic tachyon vacuum solution.

\section{Identity-based solutions in bosonic open SFT}

The identity-based solution can be expressed as [4, 6, 7, , 8,

$$
\Psi_{0}=Q_{L}\left(e^{h}-1\right) I-C_{L}\left((\partial h)^{2} e^{h}\right) I,
$$

where $I$ is the identity string field associated with the star product, and the half string operators $Q_{L}$ and $C_{L}$ are defined using the BRST current $J_{B}(z)$

\footnotetext{
${ }^{1}$ There are other attempts to construct analytic solutions in SFT. See, for example, [5] and references therein.
} 
and ghost field $c(z)$ as follows,

$$
Q_{L}(f)=\int_{C_{\text {left }}} \frac{d z}{2 \pi i} f(z) J_{B}(z), \quad C_{L}(g)=\int_{C_{\text {left }}} \frac{d z}{2 \pi i} g(z) c(z) .
$$

Here, $C_{\text {left }}$ denotes the contour along a right semi-unit circle from $-i$ to $i$, which conventionally corresponds to the left half of strings. The function $h(z)$ is defined on the whole unit circle. For the function $h(z)$ satisfying $h(-1 / z)=$ $h(z)$ and $h( \pm i)=0$, the equations of motion, $Q_{B} \Psi_{0}+\Psi_{0} * \Psi_{0}=0$, hold.

Hereafter, we consider the identity-based solution derived from the function

$$
\begin{aligned}
h(z) & =\log \left(1+\frac{a}{2}\left(z+\frac{1}{z}\right)^{2}\right) \\
& =-\log (1-Z(a))^{2}-\sum_{n=1}^{\infty} \frac{(-1)^{n}}{n} Z(a)^{n}\left(z^{2 n}+z^{-2 n}\right),
\end{aligned}
$$

where $Z(a)=(1+a-\sqrt{1+2 a}) / a$. For the solution to satisfy the reality condition, the parameter $a$ is larger than or equal to $-1 / 2$. This function gives the simplest example to capture typical feature of the identity-based solution.

i) It has a well-defined universal Fock space expression: if we expand the solution by Fock space states

$$
\left|\Psi_{0}(a)\right\rangle=\varphi_{0}(a) c_{1}|0\rangle+v_{0}(a) c_{1} L_{-2}^{X}|0\rangle+u_{0}(a) c_{-1}|0\rangle+\cdots
$$

the coefficients $\varphi_{0}(a), v_{0}(a)$ and $u_{0}(a)$ are finite for all $a$.

ii) For $a>-1 / 2$, the solution can be expressed as a pure gauge form connecting to a trivial configuration, but at $a=-1 / 2$, the solution can be given as a type of singular gauge transformation of the trivial configuration [4]: we can rewrite the solution for generic $a$ as

$$
\Psi_{0}(a)=g(a) * Q_{B} g^{-1}(a),
$$

but $g(a)$ becomes singular at $a=-1 / 2$.

These facts suggest that the solution for $a>-1 / 2$ is a trivial pure gauge, but it becomes a non-trivial configuration at $a=-1 / 2$. 
Moreover, if we expand the string field as $\Psi=\Psi_{0}+\Phi$, we can obtain the action for the fluctuation around the identity-based solution. In the expanded theory, the kinetic operator can be written as

$$
\begin{aligned}
Q^{\prime}= & (1+a) Q_{B}+\frac{a}{2}\left(Q_{2}+Q_{-2}\right)+4 a Z(a) c_{0}-2 a Z(a)^{2}\left(c_{2}+c_{-2}\right) \\
& -2 a\left(1-Z(a)^{2}\right) \sum_{n=2}^{\infty}(-1)^{n} Z(a)^{n-1}\left(c_{2 n}+c_{-2 n}\right),
\end{aligned}
$$

where we expand the BRST current and ghost field as $J_{B}(z)=\sum_{n} Q_{n} z^{-n-1}$ and $c(z)=\sum_{n} c_{n} z^{-n+1}$, respectively.

The following facts have been known for the expanded theory for $a>-1 / 2$ :

1. The action obtained by expanding around the solution can be transformed back to the action with the original BRST charge [4].

2. The new BRST charge gives rise to the cohomology, which has one-to-one correspondence to the cohomology of the original BRST charge [6].

3. The expanded theory reproduces ordinary open string amplitudes 8 .

These are consistent with the expectation that the solution for $a>-1 / 2$ corresponds to a trivial pure gauge. On the other hand, we find completely different properties in the expanded theory around the solution with $a=-1 / 2$ :

4. The new BRST charge has vanishing cohomology in the Hilbert space with the ghost number one 6 .

5. The open string scattering amplitudes vanish and the result is consistent with the absence of open string excitations [8].

From these facts, it would be reasonable to expect that the identity-based solution at $a=-1 / 2$ indeed corresponds to the tachyon vacuum solution. Hence, we expect that the identity-based solution corresponds to a trivial pure 
gauge form for almost all the parameter region and it can be regarded as the tachyon vacuum solution at $a=-1 / 2$.

To prove our conjecture, we have to calculate the vacuum energy of the identity-based solution directly. Formally, the vacuum energy can be calculated as

$$
V\left(\Psi_{0}(a)\right)=(\text { matter sector }) \times(\text { ghost sector })=\infty \times 0,
$$

namely, the vacuum energy is given as an indefinite quantity. To calculate the vacuum energy, it is necessary to apply a kind of canonical regularization to fix the ambiguity. Indeed, the level can be regarded as a regularization parameter for the numerical solution in the Siegel gauge. Hence, the difficulty of calculating the vacuum energy seems to arise from the lack of such a regularization method for the identity-based solution.

However, we can provide indirect evidence that supports the possibility of calculating the vacuum energy. The vacuum structure in the theory expanded around the identity-based solution has been analyzed using level truncation approximation and then we have found the following results:

6. A numerical analysis shows that the nonperturbative vacuum found for $a>-1 / 2$ disappears as $a$ approaches $-1 / 2$ [7].

7. The energy of the nonperturbative vacuum for $a>-1 / 2$ becomes closer to the value appropriate to cancel the D-brane tension as the truncation level increases [7].

These imply that the theory around the identity-based solution for $a>-1 / 2$ has the tachyon vacuum, but the theory at $a=-1 / 2$ is stable. From consistency with the theory before expanding a string field, it follows that the vacuum energy of the identity-based solution itself is zero for $a>-1 / 2$ and it is equal to the tachyon vacuum energy at $a=-1 / 2$. 


\section{Annihilation of tachyon vacuum}

We consider the tachyon vacuum in the expanded theory around the identitybased solution. The expanded theory has a gauge symmetry under

$$
\delta \Phi=Q^{\prime} \Lambda+\Phi * \Lambda-\Lambda * \Phi
$$

where $Q^{\prime}$ is given by Eq. (77). To find classical solutions in the theory, we impose the Siegel gauge condition on the fluctuation string field; $b_{0} \Phi=0$. Under the Siegel gauge condition, the potential can be expressed as

$$
f_{a}(\Phi)=2 \pi^{2}\left(\frac{1}{2}\left\langle\Phi, c_{0} L(a) \Phi\right\rangle+\frac{1}{3}\langle\Phi, \Phi * \Phi\rangle\right),
$$

where it is normalized as -1 for the tachyon vacuum solution at $a=0$. Here, the operator $L(a)$ is given by

$$
L(a)=(1+a) L_{0}+\frac{a}{2}\left(L_{2}+L_{-2}\right)+a\left(q_{2}-q_{-2}\right)+4(1+a-\sqrt{1+2 a}),
$$

where $L_{n}$ is a total Virasoro generator and $q_{n}$ is a mode of the ghost number current 2

If the identity-based solution corresponds to a trivial pure gauge for $a>$ $-1 / 2$ and then to the tachyon vacuum for $a=-1 / 2$, the potential (10) should be illustrated as in Fig. 1, For $a>-1 / 2$, the tachyon vacuum configuration $\Phi_{1}$ should minimize the potential, since the expanded theory for $\Phi$ is still the theory on the perturbative open string vacuum. However, at $a=-1 / 2$, the trivial configuration $\Phi=0$ should be stable since the expanded theory is expected to be already on the tachyon vacuum. In addition, the theory at $a=-1 / 2$ should have an unstable solution corresponding to the perturbative open string vacuum of the unexpanded original theory.

Let us suppose that we obtain the tachyon vacuum solution $\Phi_{1}$ in the expanded theory and we evaluate its vacuum energy. Then, the vacuum energy

${ }^{2}$ The expression is derived from $L(a)=\left\{Q^{\prime}, b_{0}\right\}$. It can be rewritten only using ghost twisted Virasoro operators as in Ref. [8]. 


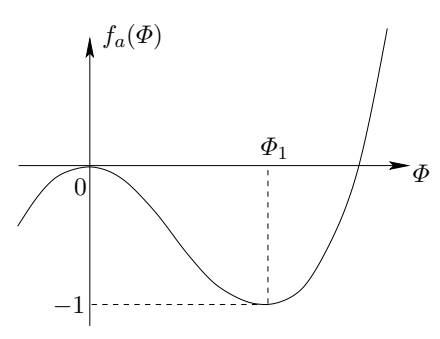

(1) $a>-1 / 2$

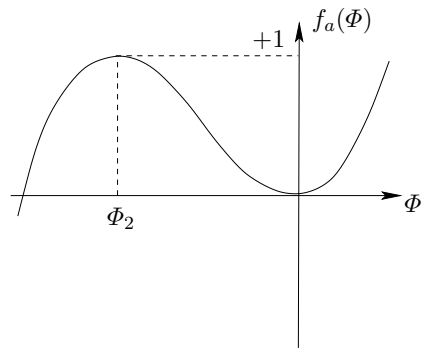

(2) $a=-1 / 2$

Figure 1: Vacuum structure expected for the theory expanded around the identity-based solution. (1) For $a>-1 / 2$, the theory should have a nontrivial vacuum solution, the vacuum energy of which cancels the D-brane tension. (2) At $a=-1 / 2$, the trivial configuration $\Phi=0$ should be stable. An unstable solution is expected to exist and its vacuum energy should be equal to the D-brane tension.

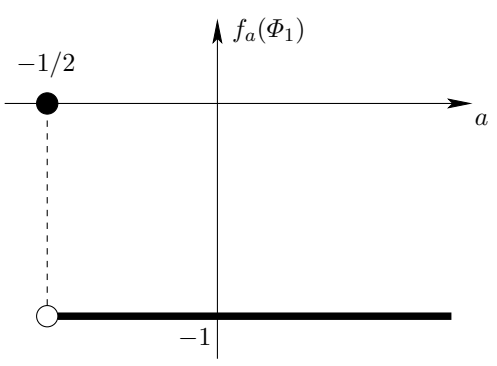

Figure 2: Vacuum energy expected for the nontrivial stable solution $\Phi_{1}$.

$f_{a}\left(\Phi_{1}\right)$ is given as a function of the parameter $a$. If the potential has such a structure as previously expected, the vacuum energy is -1 for $a>-1 / 2$ but the solution $\Phi_{1}$ becomes trivial and therefore its vacuum energy annihilates at $a=-1 / 2$ (see Fig. 2).

Now, let us consider the stable solution $\Phi_{1}$ by level truncation calculation to confirm the above conjecture. Since the level truncation is a good approximation, the vacuum energy for the truncated solution is considered to approach the step function in Fig. 2 as the truncation level is increased. We apply an iterative approximation algorithm as used in Refs. [9] and [10] to find the stable 
solution.

We show plots of the vacuum energy for the resulting solution in Fig. 3 We can find that, for various $a$, the resulting plots approach the tachyon vacuum energy -1 as the truncation level is increased. Then, for decreasing $a$ to $-1 / 2$, the vacuum energy increases rapidly to zero from -1 . As a whole, the plots become closer to the step function as depicted in Fig. 2 as the truncation level increases. These results support our expectation for the vacuum structure associated with the stable solution $\Phi_{1}$.

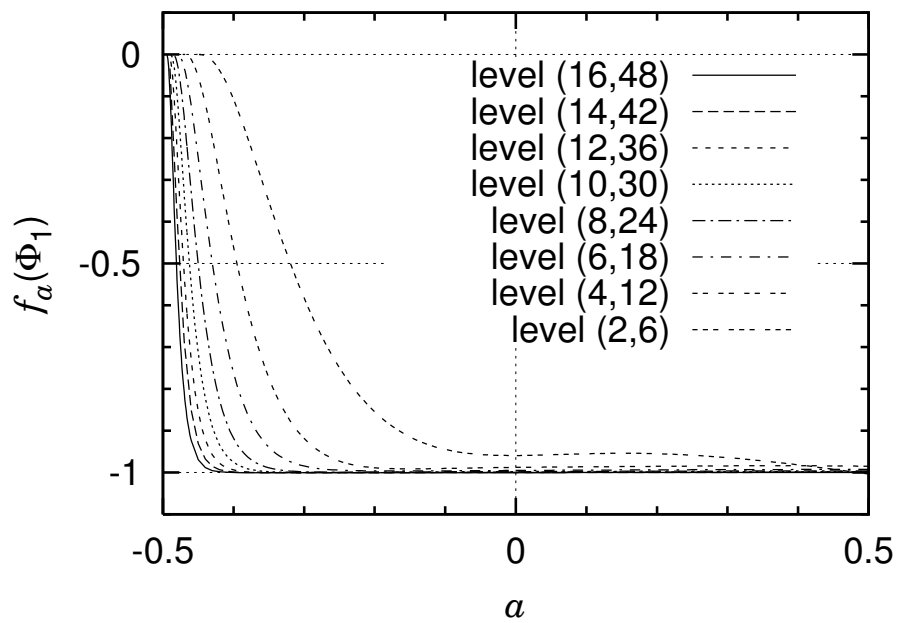

Figure 3: Vacuum energy of the numerical stable solutions in the expanded theory around the identity-based solution. As the truncation level is increased, the resulting plots approach the step function expected as in Fig. 2

\section{Emergence of unstable vacuum}

We consider an unstable solution in the theory expanded around the identitybased solution. For $a>-1 / 2$, the expanded theory is unstable at $\Phi=0$. However, the expanded theory is expected to be already on the tachyon vacuum for $a=-1 / 2$; namely, $\Phi=0$ is a stable vacuum at $a=-1 / 2$. If that is the 
case, the expanded theory for $a=-1 / 2$ should have a nontrivial unstable solution corresponding to the perturbative vacuum in the original theory.

Since the unstable solution $\Phi_{2}$ should correspond to the perturbative open string vacuum, the vacuum energy of the unstable solution should be equal to the D-brane tension (not the minus D-brane tension). Therefore, the vacuum energy of $\Phi_{2}$ is expected to behave as depicted in Fig. 4. $f_{a}\left(\Phi_{2}\right)$ is trivially zero for $a>-1 / 2$, but it should be increased to +1 owing to the emergence of the unstable solution at $a=-1 / 2$.

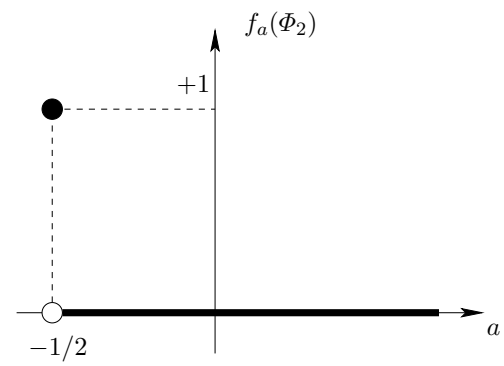

Figure 4: Vacuum energy expected for the unstable solution $\Phi_{2}$.

The vacuum energy and the gauge invariant overlap for $\Phi_{2}$ at $a=-1 / 2$ are shown in Table. 1. We find that the vacuum energy approaches the expected value of +1 as the truncation level is increased. At level $(20,60)$, the vacuum energy is about $19 \%$ over, although it is about $260 \%$ at level $(2,6)$. Moreover, the gauge invariant overlap also takes around the expected value of -1 . These results suggest that the level truncation approximation is also applicable to the analysis of the unstable solution. Then, it is reasonably confirmed that the unstable solution does exist as expected in the expanded theory at $a=-1 / 2$.

Let us consider the unstable solution $\Phi_{2}$ for various $a(>-1 / 2)$. The resulting vacuum energy of the unstable solution is depicted in Fig. 5. The vacuum energy is around the expected value for $a=-1 / 2$, but it decreases rapidly to 
zero for increasing $a 3$ Thus, we find that the vacuum energy of the unstable solution approaches the step function as expected in Fig. 4 for increasing truncation levels.

Table 1: Vacuum energy and gauge invariant overlap for the unstable solution $\Phi_{2}$ at $a=-1 / 2$.

\begin{tabular}{|c|c|c|}
\hline level & vacuum energy & gauge inv. overlap \\
\hline \hline$(0,0)$ & 2.3105795 & -1.0748441 \\
\hline$(2,6)$ & 2.5641847 & -1.0156983 \\
\hline$(4,12)$ & 1.6550774 & -0.9539832 \\
\hline$(6,18)$ & 1.6727496 & -0.9207572 \\
\hline$(8,24)$ & 1.4193393 & -0.9377548 \\
\hline$(10,30)$ & 1.4168893 & -0.9110994 \\
\hline$(12,36)$ & 1.3035715 & -0.9237917 \\
\hline$(14,42)$ & 1.2986472 & -0.9056729 \\
\hline$(16,48)$ & 1.2357748 & -0.9229035 \\
\hline$(18,54)$ & 1.2310583 & -0.9086563 \\
\hline$(20,60)$ & 1.1915648 & -0.9212376 \\
\hline
\end{tabular}

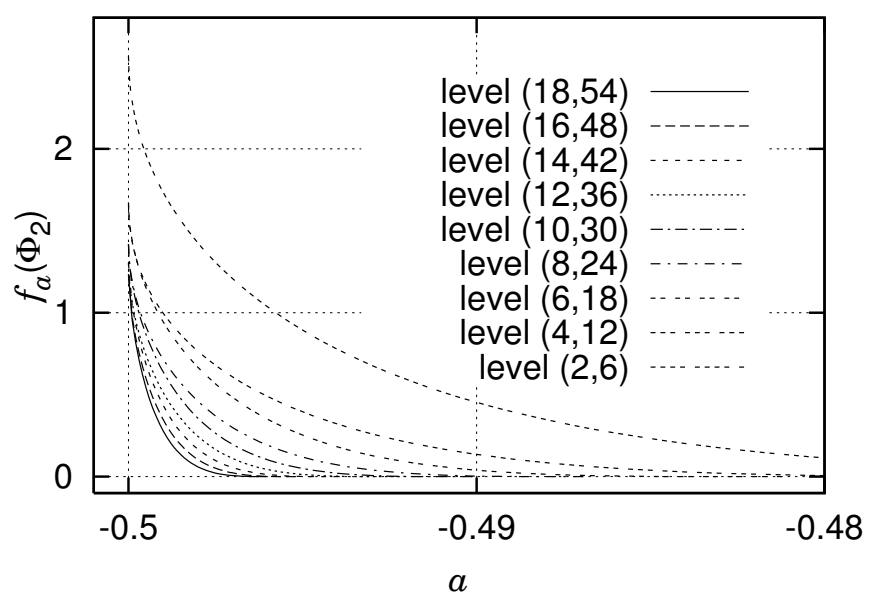

Figure 5: Vacuum energy of the unstable solution for various $a$.

\footnotetext{
${ }^{3}$ Note that in Fig. 5 the parameter $a$ ranges from -0.5 to -0.48 and this range is much narrower than that of Fig. 3
} 


\section{$5 \quad$ Summary and discussion}

We have found that the stable and unstable solutions in the Siegel gauge, $\Phi_{1}$ and $\Phi_{2}$, numerically exist in the theory expanded around the identity-based solution. For these solutions, we have evaluated the vacuum energy in terms of level truncation approximation up to level $(20,60)$. In our paper [5], we have also evaluated the gauge invariant overlap for these solutions. As the truncation level is increased, the plots of the gauge invariants for the parameter $a$ become remarkably closer to the behavior expected from the vacuum structure in Fig. 1. These results strongly support our expectation that the identity-based solution corresponds to a trivial pure gauge configuration for $a>-1 / 2$, but it can be regarded as the tachyon vacuum solution for $a=-1 / 2$.

While these results are encouraging, the direct calculation remains one of the most difficult issues in string field theory. However, the success of the numerical analysis is a characteristic feature of the identity-based solution. In addition to the Schnabl-type solutions, the identity-based solution seems to provide complementary approaches to a deeper understanding of the string field theory. In particular, we expect that the worldsheet picture in the expanded theory clarifies the existence of closed strings on the tachyon vacuum [11, 12.

\section{Acknowledgements}

This work was supported in part by JSPS Grant-in-Aid for Scientific Research (C) (\#21540269). The work of I. K. was supported in part by a Special Postdoctoral Researchers Program at RIKEN. The work of T. T. was supported in part by Nara Women's University Intramural Grant for Project Research. Numerical computations in this work were partly carried out on the Computer Facility of the Yukawa Institute for Theoretical Physics in Kyoto University and the RIKEN Integrated Cluster of Clusters (RICC) facility. 


\section{References}

[1] A. Sen and B. Zwiebach, J. High Energy Phys. 03 002, (2000) arXiv:hep-th/9912249.

[2] M. Schnabl, Adv. Theor. Math. Phys. 10 433, (2006) arXiv:hep-th/0511286.

[3] I. Ellwood and M. Schnabl, J. High Energy Phys. 02 096, (2007) arXiv:hep-th/0606142.

[4] T. Takahashi and S. Tanimoto, J. High Energy Phys. 03 033, (2002) arXiv:hep-th/0202133.

[5] I. Kishimoto and T. Takahashi, Prog. Theor. Phys. 122 385, (2009) arXiv:0904.1095 [hep-th]].

[6] I. Kishimoto and T. Takahashi, Prog. Theor. Phys. 108 591, (2002) arXiv:hep-th/0205275.

[7] T. Takahashi, Nucl. Phys. B 670 161, (2003) arXiv:hep-th/0302182.

[8] T. Takahashi and S. Zeze, Prog. Theor. Phys. 110 159, (2003) arXiv:hep-th/0304261].

[9] D. Gaiotto and L. Rastelli, J. High Energy Phys. 08 048, (2003) arXiv:hep-th/0211012.

[10] I. Kishimoto and T. Takahashi, Prog. Theor. Phys. 121 695, (2009) [arXiv arXiv:0902.0445].

[11] N. Drukker, J. High Energy Phys. 08 017, (2003) arXiv:hep-th/0301079.

[12] Y. Igarashi, K. Itoh, F. Katsumata, T. Takahashi and S. Zeze, Prog. Theor. Phys. 114 1269, (2006) arXiv:hep-th/0506083. 